\title{
Ankle arthroscopy in children
}

\author{
Nijil Val Vasukutty ${ }^{1}$, Hawar Akrawi², Bipin Theruvil ${ }^{3}$, Mike Uglow ${ }^{2}$
}

${ }^{1}$ Pilgrim Hospital, Boston, UK

${ }^{2}$ Southampton General Hospital, Southampton, UK

${ }^{3}$ Royal Bournemouth Hospital, Bournemouth, UK

\begin{abstract}
INTRODUCTION Arthroscopy of the ankle has gained acceptance as a procedure for diagnosing and treating chronic and posttraumatic ankle problems. The senior author's perception is that magnetic resonance imaging (MRI) under-diagnoses anterior soft tissue impingement lesions in children. The purpose of this study was retrospectively to analyse the outcome of ankle arthroscopy in the paediatric age group.

PATIENTS AND METHODS Between March 2005 and September 2007, 23 children underwent ankle arthroscopy for post-traumatic pathology. The indications for arthroscopy were failure of non-operative treatment for at least 12 weeks or a grade 3 or 4 osteochondral defect (OCD) on imaging.

RESULTS At arthroscopy, OCDs were visualised in 12 cases and impingement lesions were seen in 17 ankles. MRI was performed in 8 of these 12 cases and only 1 suggested the possibility of an impingement lesion. Of the 17 cases of impingement seen on arthroscopy, 12 reported mechanical symptoms preoperatively and 4 were unstable on examination under anaesthetic. Eighteen of the twenty-three patients had complete relief of symptoms at 3 months. Eighteen children who were evaluated at one-year follow up had a mean American Orthopaedic Foot and Ankle Society score of 87. 5 (range: 49-100).

CONCLUSIONS Ankle arthroscopy has a successful outcome in paediatric patients and the results are comparable with those reported in adult series. MRI was found to be insensitive for the diagnosis of soft tissue impingement of the ankle.
\end{abstract}

\section{KEYWORDS}

Ankle arthroscopy - Children's orthopaedics - Talar osteochondral defects - MRI accuracy compared with arthroscopy

Accepted 23 December 2010

\section{CORRESPONDENCE TO}

Mike Uglow, Consultant Orthopaedic Surgeon, Southampton General Hospital, Tremona Road, Southampton, Hampshire SO16 6YD, UK

E: mike.uglow@suht.swest.nhs.uk

Approximately 1 in 10,000 people per day suffer an ankle injury. ${ }^{1}$ In athletes this number can be as high as 5.23 ankle injuries per 10,000 athlete exposures..$^{2}$ Fong et al have published a systematic review in which they concluded that ankle injuries were the most common type of injury in 24 out of 70 sports, with ankle sprain being the most frequent. ${ }^{3}$ The majority of patients are treated conservatively and the outcome is usually satisfactory. It is reasonable to allow a period of time for the soft tissue injury to settle down completely before returning to sport. However, a subset of patients may continue to experience pain after what appears to be a routine sprain.

Such persistent pain may be due to a number of conditions including insufficient rehabilitation, post-traumatic impingement syndrome, occult osteochondral defects (OCDs), trauma to the peroneal tendon or syndesmosis, chronic instability or reflex sympathetic dystrophy. The evaluation of such persistent ankle pain should involve a thorough clinical evaluation, preferably by a surgeon who has a special interest in foot and ankle surgery, supplemented by advanced imaging modalities like magnetic resonance imaging (MRI) where required.
Over the last 30 years arthroscopy of the ankle has gained acceptance as a procedure in diagnosing and treating chronic and post-traumatic ankle problems. ${ }^{4}$ Arthroscopy of the ankle should be performed after all non-operative approaches have failed except in the treatment of displaced osteochondral lesions. ${ }^{5}$ The diagnostic indications include pain, swelling, stiffness, instability and locking. An additional indication would be a negative workup in a patient with significant ankle symptoms unresponsive to conservative management. ${ }^{6}$ Often, chondral fractures and soft tissue lesions undetected on clinical and radiological evaluation and MRI may become obvious on arthroscopic examination.

\section{Patients and Methods}

This was a retrospective clinical review of 23 children who had undergone arthroscopic surgery for ankle pathology at our institution between March 2005 and September 2007. The senior author (MU) performed all the surgical procedures. After a clinical examination by the senior author or an experienced trainee, all patients had standard radiographs consisting of anteroposterior standing and true lateral views 


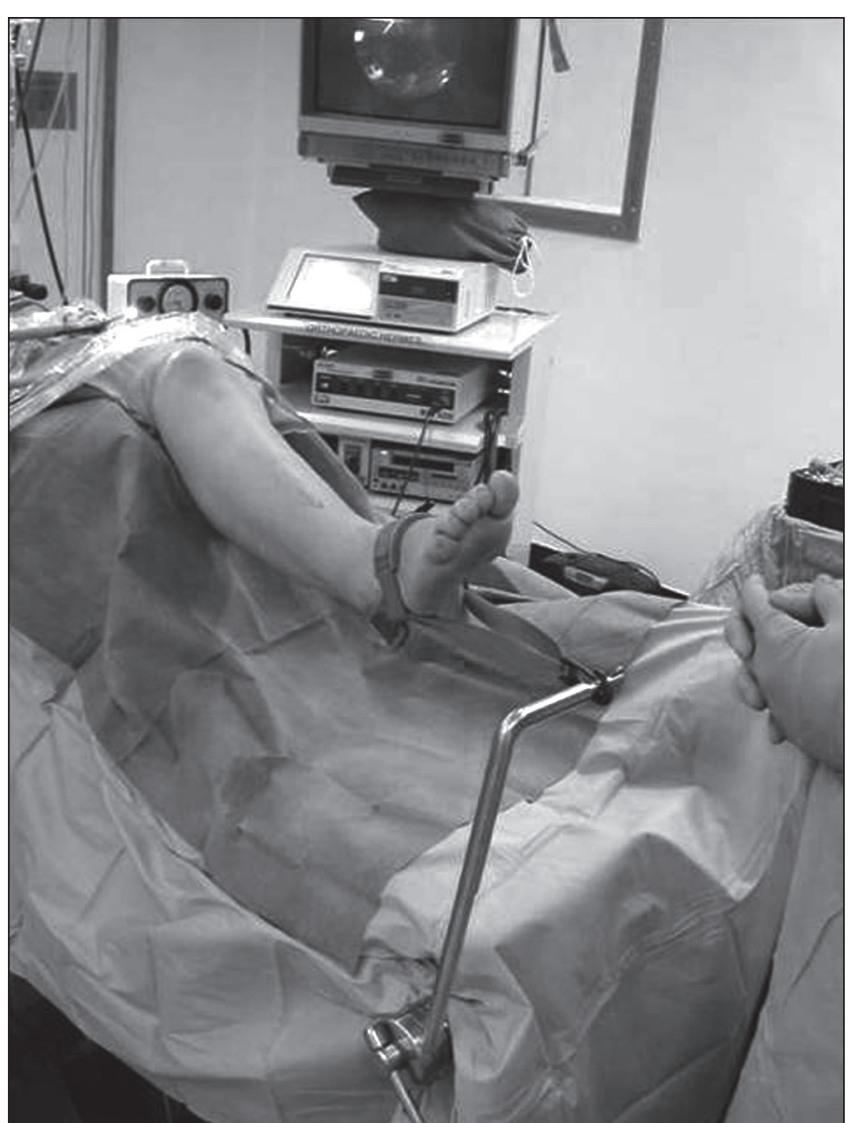

Figure 1 Arthroscopy was performed using a tourniquet and a non-invasive ankle distractor.

of the ankle. Twelve children had MRI preoperatively. Our indication for MRI was presence of mechanical symptoms like locking and/or instability with a normal plain radiograph. It is not easy to do a proper clinical examination in children with painful ankles in an outpatient setting. Whenever possible, an ankle examination was performed. An examination under anaesthetic (EUA) was always performed prior to surgery. Postoperatively, the patients were reviewed at 6 weeks. Patients were discharged in cases where symptoms had become unobtrusive or absent.

\section{Operative procedure}

Our inclusion criteria were non-responsiveness to conservative treatment, including physiotherapy, for at least 12 weeks or a Berndt and Harty grade 3 or 4 OCD on imaging. ${ }^{7}$ After an EUA, arthroscopy was performed with the patient supine, using a leg holder. A tourniquet and a non-invasive ankle distractor were used (Fig 1). The joint was visualised through standard anteromedial and anterolateral portals. All soft tissue lesions were debrided with a $2.9 \mathrm{~mm}$ or $3.5 \mathrm{~mm}$ shaver. Superficial chondral flaps were debrided. These included loose edges of grade 2 OCD lesions. All grade 3 or 4. OCDs were curetted down to fresh cancellous bone, ensuring vertical edges to the defect. The portals were closed with a single nylon suture.
All patients were given wool and crepe dressings for 24 hours. This was reduced to an adhesive dressing and elastic support bandage, and after 2 weeks the wound was reviewed and the sutures were removed. All patients were mobilised with partial weight bearing crutches for comfort but allowed to increase to full weight bearing as pain allowed. Physiotherapy was utilised to enhance range of motion and proprioception when required.

\section{Results}

There were 14 boys and 9 girls with an average age of 13.5 years (range: 8.6-18 years). Thirteen children presented acutely. For those who presented late, the average duration of symptoms was 17.8 months (range: 2-36 months). The most common complaint was pain while walking or with sports (22 out of 23 children). Nine children reported mechanical symptoms in the form of instability or clicking, seven children attributed their ankle problems to sport injuries, three had falls from a trampoline and seven others had simple falls sustaining inversion injuries. Six patients, one of whom was a keen dancer, could not recollect any specific instance of trauma. Twenty-two out of twenty-three children presented with pain and seven $(30 \%)$ had associated instability. Nine (39\%) children had swelling of varying degrees and five $(22 \%)$ reported recurrent episodes of locking. Four out of twenty-three $(17 \%)$ children showed restricted range of movement. Nine (39\%) children had deep ankle pain, six (26\%) had signs of anterolateral impingement described by Molloy et $a l,{ }^{8}$ while none showed clinical features of posterior impingement. Tenderness over the syndesmoses could be elicited in $8(35 \%)$ cases. No varus or valgus malalignment was observed.

Plain $x$-rays showed OCDs in five patients. Of the twelve preoperative MRI scans, five showed OCDs, two showed evidence of tarsal coalition (one subtalar and one calcaneo-navicular), one showed features suggestive of posterior ankle impingement and one showed evidence of lateral ligament injury. The remaining three scans were normal. Preoperative EUA showed instability in the form of a positive anterior drawer or forced inversion test in seven $(30 \%)$ ankles. In these unstable ankles, three had OCDs and four had associated impingement lesions.

There were a total of 15 (65\%) OCDs (Fig 2) in the series. There were three cases in which MRI had reported an OCD and the cartilage was found to be intact on arthroscopy, confirming these to be grade 1 OCDs. Arthroscopic findings identified two grade 4 lesions, three grade 3 and seven grade 2 lesions.

The three with grade 1 lesions were found to have anterior impingement lesions and, of the remaining twelve with chondral involvement, six cases were in isolation and six had impingement lesions in addition to the OCD.

Impingement lesions (Fig 3) were seen in 17 (74\%) ankles. Nine of these were anterolateral, one was anteromedial and two were syndesmotic. Five showed more generalised synovitis, which was considered to be impinging.

Correlating the presenting symptoms with arthroscopic findings, seven out of nine children who presented with me- 


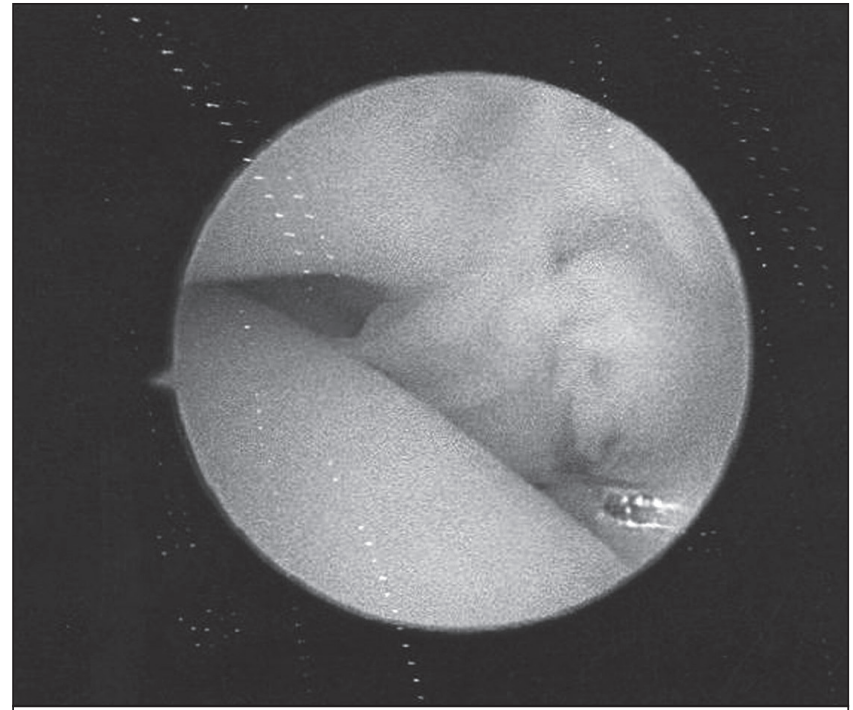

Figure 2 Osteochondral defect

chanical symptoms (instability or locking) had impingement lesions at arthroscopy and the remaining two had OCDs. Out of the nine patients with anterolateral impingement lesions, six showed clinical signs on preoperative evaluation. Although syndesmotic tenderness was elicited in eight cases, only two of these showed impingement lesions in this location. Of the seven ankles that were unstable on EUA, three showed OCDs and four showed impingement lesions on arthroscopy. It was interesting to note that there were six ankles with combined OCDs and impingement lesions that were stable on EUA.

Eighteen of the twenty-three patients $(78 \%)$ had complete relief of symptoms at 3 months with a mean American Orthopaedic Foot and Ankle Society score of 87 . They returned to their normal activity and all seven of our patients involved in competitive sport were able to return to sports. Five children had ongoing symptoms at 6 months. Two children had persisting pain with instability, two had instability without pain and one reported pain without any mechanical symptoms. One case with painful instability, and that had impingement on initial arthroscopy, required a Broström ligament repair. One case needed a repeat arthroscopy and debridement at 12 months, which relieved her symptoms. This child had an OCD on initial assessment that was debrided. The remaining three were advised restricted activity for 6 months, which relieved their symptoms. Of the patients who were treated with debridement for impingement, all but one had their symptoms completely resolved. The one remaining patient required a further arthroscopy and was found to have developed additional soft tissue impingement at the site of the lateral portal. This was successfully debrided with complete resolution. The senior author has experienced this occurrence in $1.7 \%$ of arthroscopic debridement procedures (personal unpublished data). Three of the four patients with persistent instability had presented with instability initially and were unstable on EUA. There was only one case of posterior impingement and this had a good outcome.

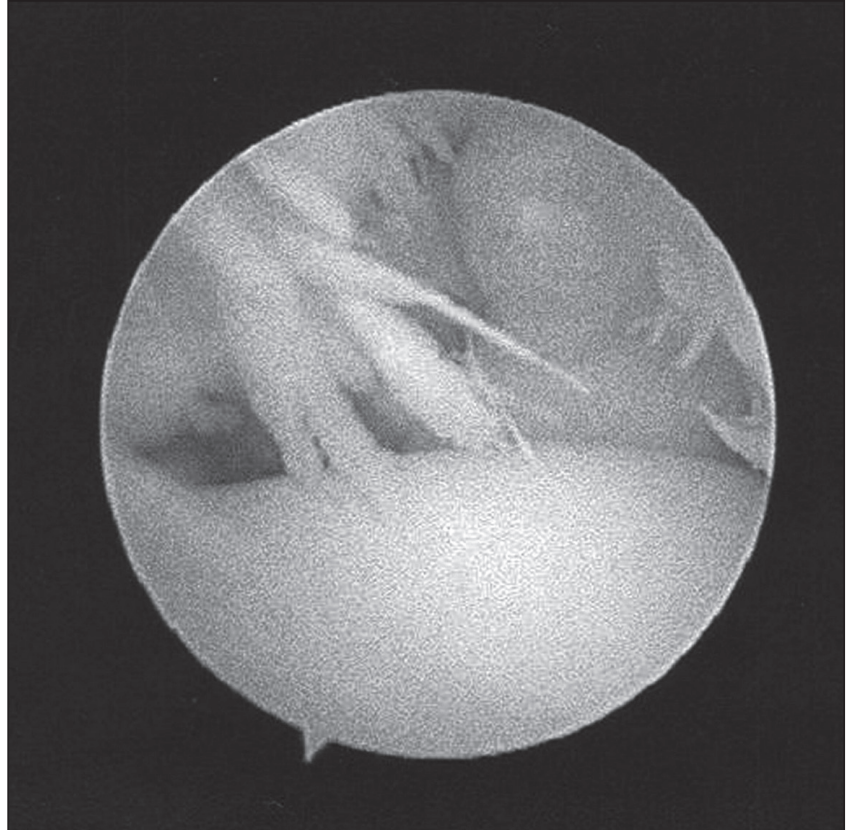

Figure 3 Impingement lesion

\section{Discussion}

This series reports the results of arthroscopic treatment of ankle injuries exclusively in the paediatric age group. The results of arthroscopic treatment of anterolateral soft tissue impingement have been reported as being uniformly successful in adults, and our results compare with those. ${ }^{9}$ We observed excellent results with complete pain relief and return to function in $78 \%$ of our cases. Ferkel et al reported good or excellent results in $85 \%$ of patients with anterolateral ankle impingement. ${ }^{10}$ In our population we treated impingement lesions both in isolation and in the presence of OCDs and found that the latter gives the impression of poorer results. Grade 1 and 2 OCDs are treated expectantly and symptoms are to be expected until revascularisation of the lesions occur.

In our series there were 7 ankles with clinical instability while 17 ankles showed features of impingement. This suggests that the most likely source of ankle pain in this patient population may be due to instability, soft tissue impingement or osteochondral defects or a combination of these. Kim came to a similar conclusion in his series of 52 adult patients who had arthroscopic treatment for similar pathology. ${ }^{11}$ Liu et al reported on a series of 55 patients with anterolateral ankle impingement in whom 14 (25\%) had a positive anterior drawer. ${ }^{12} 87 \%$ had good to excellent results following arthroscopic treatment. A distal fascicle of the anteroinferior tibiofibular ligament (Bassett's ligament) has been described as being a cause for anterior impingement ${ }^{13}$ but we have not observed this in any of our cases.

Only one patient in our series had persisting problems from instability and subsequently required a lateral ligament reconstruction after the initial debridement. We feel that symptoms of instability could be due to impingement 
and can be improved with debridement and proprioceptive physiotherapy. Hence, in cases with instability, our approach is to perform an arthroscopy with a view to debridement in the first instance after failure of non-operative means to control symptoms.

MRI is stated as being an important adjunct for assessment of injury and post-traumatic pathology and some authors have reported $100 \%$ accuracy for this imaging modality. ${ }^{14,15}$ Ultrasound imaging has also been used to diagnose post-traumatic ankle pathology with good results. ${ }^{16}$ However, in our series there was poor correlation between findings on MRI and intraoperative appearance at arthroscopy. There were two cases where preoperative scans failed to pick up small chondral defects. In our series, 17 cases had impingement and, of these, 12 had preoperative MRI. However, the imageing correctly identified the impingement lesion in only one case. In the adult population a magnetic resonance arthrogram increases the diagnostic accuracy of soft tissue lesions but this would be considered inappropriate for the majority of children.

\section{Conclusions}

The experience gained from this series confirms the importance of a through clinical examination to assess ligament stability and the presence or absence of impingement. Radiological investigation to augment the examination findings should include plain radiographs as well as MRI, which is sensitive for identifying osteochondral defects but not for impingement. The diagnosis of impingement is essentially a clinical diagnosis. At short- to intermediate-term follow up, we feel that arthroscopic assessment and debridement is an effective way of treating post-traumatic ankle pathology, particularly soft tissue impingement and OCDs in children.

\section{References}

1. O'Loughlin PF, Heyworth BE, Kennedy JG. Current concepts in the diagnosis and treatment of osteochondral lesions of the ankle. Am J Sports Med 2010; 38: 392-404.

2. Nelson AJ, Collins CL, Yards EE et al. Ankle injuries among United States high school athletes, 2005-2006. J Athl Train 2007; 42: 381-387.

3. Fong DT, Hong Y, Chan LK et al. A systematic review on ankle injury and ankle sprain in sports. Sports Med 2007; 37: 73-94.

4. van Dijk CN, van Bergen CJ. Advancements in ankle arthroscopy. J Am Acad Orthop Surg 2008; 16: 635-646.

5. Higuera J, Laguna R, Peral M et al. Osteochondritis dissecans of the talus during childhood and adolescence. J Pediatr Orthop 1998; 18: 328-332.

6. Stetson WB, Ferkel RD. Ankle arthroscopy: ii. indications and results. J Am Acad Orthop Surg 1996; 4: 24-34.

7. Benthien RA, Sullivan RJ, Aronow MS. Adolescent osteochondral lesion of the talus. Ankle arthroscopy in pediatric patients. Foot Ankle Clin 2002; 7 651-667.

8. Molloy S, Solan MC, Bendal SP. Synovial impingement in the ankle. A new physical sign. J Bone Joint Surg Br 2003; 85: 330-333.

9. Amendola A, Petrik J, Webster-Bogaert S. Ankle arthroscopy: outcome in 79 consecutive patients. Arthroscopy 1996; 12: 565-573.

10. Ferkel RD, Karzel RP, Del Pizzo W et al. Arthroscopic treatment of anterolateral impingement of the ankle. Am J Sports Med 1991; 19: 440-446.

11. Kim SH, Ha KI. Arthroscopic treatment for impingement of the anterolateral soft tissues of the ankle. J Bone Joint Surg Br 2000; 82: 1,019-1,021.

12. Liu SH, Raskin A, Osti L et al. Arthroscopic treatment of anterolateral ankle impingement. Arthroscopy 1994; 5: 215-218.

13. Bassett FH 3rd, Gates HS 3rd, Billys JB et al. Talar impingement by the anteroinferior tibiofibular ligament. A cause of chronic pain in the ankle after inversion sprain. J Bone Joint Surg Am 1990; 72: 55-59.

14. Robinson P, White LM, Salonen DC et al. Anterolateral ankle impingement: MR arthrographic assessment of the anterolateral recess. Radiology 2001; 221: $186-190$.

15. Jordan LK 3rd, Helms CA, Cooperman AE, Speer KP. Magnetic resonance imaging findings in anterolateral impingement of the ankle. Skeletal Radiol 2000; 29: 34-39.

16. McCarthy CL, Wilson DJ, Coltman TP. Anterolateral ankle impingement: findings and diagnostic accuracy with ultrasound imaging. Skeletal Radiol 2008; 37: 209-16. 